\title{
Clinical significance of enhancer of zeste homolog 2 and histone deacetylases 1 and 2 expression in peripheral T-cell lymphoma
}

\author{
HUILAI ZHANG $^{1 *}$, HUIJUAN LV ${ }^{1 *}$, XIAOHUI JIA ${ }^{1 *}$, GE HU $^{1}$, LINGZHE KONG ${ }^{1}$, TINGTING ZHANG ${ }^{1}$, \\ LINYU LI ${ }^{1}$, YI PAN ${ }^{2}$, QIONGLI ZHAI ${ }^{2}$, BIN MENG $^{2}$, XI WANG $^{3}$, HUAQING WANG $^{4}$ and XIANHUO WANG ${ }^{*}$ \\ Departments of ${ }^{1}$ Lymphoma and ${ }^{2}$ Pathology, Sino-US Center for Lymphoma and Leukemia, Tianjin Medical \\ University Cancer Institute and Hospital, National Clinical Research Center of Cancer, Key Laboratory of \\ Cancer Prevention and Therapy, Tianjin 300060; ${ }^{3}$ Department of Cell Biology, College of Basic Medical \\ Sciences, Tianjin Medical University, Tianjin 300070; ${ }^{4}$ Department of Oncology, Tianjin Union \\ Medical Center, The Affiliated Hospital of Nankai University, Tianjin 300121, P.R. China
}

Received November 27, 2016; Accepted March 19, 2019

DOI: $10.3892 / \mathrm{ol} .2019 .10410$

\begin{abstract}
Epigenetics serve a key role in peripheral $\mathrm{T}$ cell lymphoma (PTCL). The purpose of the present study was to investigate the clinical significance of enhancer of zeste homolog 2 (EZH2) and histone deacetylase 1 and 2 (HDAC1/2) expression in PTCL. A total of 82 patients were enrolled in the present study, including 43 with PTCL not otherwise specified (PTCL-NOS), 10 with angioimmunoblastic T-cell lymphoma (AITL), 14 with natural killer/T-cell lymphoma (NK/TCL) and 15 with anaplastic large cell lymphoma (ALCL). EZH2 and HDAC1/2 expression was detected by immunohistochemistry and any correlations between them were evaluated. Additionally, any correlations between EZH 2 or HDAC1/2 expression and a number of clinicopathological characteristics were analyzed, and survival curves were created. Results revealed that 55.8\% of patients with PTCL-NOS, $57.1 \%$ of patients with NK/TCL, $86.7 \%$ of patients ALCL and $50 \%$ of patients with AITL highly expressed HDAC1. Furthermore, $58.1 \%$ of patients with PTCL-NOS, $57.1 \%$ of patients with NK/TCL, $53.3 \%$ of patients with ALCL and $60 \%$ of patients with AITL highly expressed HDAC2. Additionally, 67.5\% of patients with PTCL-NOS, $50 \%$ of patients with NK/TCL, $73.3 \%$ of patients with ALCL and $60 \%$ of patients with AITL highly expressed EZH2. EZH2 expression was significantly correlated with the presence of
\end{abstract}

Correspondence to: Dr Huilai Zhang or Dr Xianhuo Wang, Department of Lymphoma, Sino-US Center for Lymphoma and Leukemia, Tianjin Medical University Cancer Institute and Hospital, National Clinical Research Center of Cancer, Key Laboratory of Cancer Prevention and Therapy, 6 Huanhuxi Road, Hexi, Tianjin 300060, P.R. China

E-mail: zhlwgq@126.com

E-mail: tjzlyy_xianhuow@163.com

*Contributed equally

Key words: T-cell lymphoma, enhancer of zeste homolog 2, histone deacetylases 1 and 2, prognosis
B symptoms, elevated LDH and elevated $\beta 2$ microglobulin (B2M; $\mathrm{P}<0.05)$, and HDAC2 expression was significantly correlated with sex, advanced clinical stages, high international prognostic index scores and elevated B2M levels $(\mathrm{P}<0.05)$ in all the patients with PTCL. However, different subtypes of PTCL are correlated with different clinical characteristics. Patients with PTCL highly expressing EZH2 or HDAC2 exhibit a poorer overall survival rate. In conclusion, $\mathrm{EZH} 2$ and $\mathrm{HDAC} 1 / 2$ were frequently upregulated in patients with PTCL, and the patients with a higher EZH2 and HDAC2 expression usually exhibited a poorer survival rate. Therefore, EZH2 and HDAC2 may be prognostic markers in patients with PTCL, particularly in those with PTCL-NOS.

\section{Introduction}

Peripheral T cell lymphoma (PTCL) is a heterogeneous disease that accounts for 20-30\% of all lymphomas in Asia (1-3). According to the World Health Organization classification (2008) (4), PTCL consists of 22 different subtypes of T-cell and NK-cell lymphomas $(5,6)$. The majority of patients with PTCL experience an aggressive disease process with a poor survival when treated with frontline therapies, and there are currently few effective treatment options. Therefore, PTCL urgently requires further research and novel treatment options in order to improve the survival of affected patients.

Epigenetics have been receiving increasing attention with respect to tumor development. Aberrant epigenetic dysregulations, including DNA methylation, histone modification, chromatin remodeling, genetic imprinting and random chromosome (X) inactivation, serve key functions in tumorigenesis. Until now, several inhibitors of histone deacetylases (HDACs), including vorinostat, panobinostat and belinostat have been reported to possess significant clinical value (7). The balance between histone acetylation and deacetylation is regulated through the opposing family of enzymes (8), histone acetylases. HDACs are critical for gene transcription and for the functions of various cellular proteins (9). The initiation and progression of a variety of tumor types have also been demonstrated to be associated with histone acetylation and deacetylation (10). Increased expression 
of HDACs reduces histone acetylation, which is widely known to occur in cancer. To date, 18 members of the HDAC family have been identified and may be categorized into four classes according to their homology, subcellular localization and enzyme reactions (11). Class I HDACs include HDACs 1, 2, 3 and 8, which are primarily responsible for regulating the acetylation of histones. HDACs enhance the interactions between histones and negatively-charged DNA by restoring the positive charge, which results in the stabilization of chromatin conformations, thereby inhibiting gene expression, particularly that of tumor suppressor genes. HDACs are overexpressed in solid tumors and hematopoietic malignancies, and contribute to disease progression and a poor prognosis (12-19). However, studies regarding the association between the HDAC expression and the prognosis or clinicopathological characteristics in PTCL are rare.

Aberrant histone methylation also serves an important role in tumorigenesis. Polycomb repressive complex 2 (PRC2), existing in distinct multiprotein complexes that bind to and modify the chromatin of target genes, methylates lysine-27 of histone H3 (H3K27) (20). PRC2 primarily consists of embryonic ectoderm development, suppressor of zeste homolog 12 , enhancer of zest homolog 2 (EZH2) and RBAP48/RBBP4 (21). H3K27 methylation may lead to inhibition of gene expression through transcriptional repression (22). EZH2, as a catalytic subunit of PRC2, serves a key role in the epigenetic silencing of target genes (23). Previous studies have revealed that the upregulation of EZH2 is associated with aggressive progression and a poor prognosis in a wide variety of tumor types (23). Certain studies on the clinical significance of EZH2 in malignant B-cell lymphoma have been reported (24). However, few studies regarding PTCL in general or its association with EZH2 have been reported.

The present study systematically studied the potential associations between HDAC or EZH2 expression and prognosis in PTCL not otherwise specified (PTCL-NOS), angioimmunoblastic T-cell lymphoma (AITL), natural killer/T-cell lymphoma (NK/TCL) and anaplastic large cell lymphoma (ALCL).

\section{Materials and methods}

Patient characteristics. A total of 82 patients with previously untreated PTCL diagnosed by a pathologist were enrolled in the present study at Tianjin Medical University Cancer Hospital (Tianjin, China) between January 2007 and December 2015. The median age of all the evaluated patients was 54 years (range, 17-80 years), with a male-to-female ratio of 1.6:1. All patients exhibited one of four subtypes, including PTCL-NOS, AITL, NK/TCL and ALCL. PTCL-NOS was the most common subtype of PTCL, accounting for 52.4\% (43/82), while AITL accounted for $12.2 \%$ (10/82), NK/TCL for $17.1 \%(14 / 82)$ and ALCL for $18.3 \%$ (15/82). Clinicopathological characteristics, including age, sex, pathological type, clinical stage (Ann Arbor-Cotswolds stage) (25), B symptoms, marrow involvement, splenomegaly, lactate dehydrogenase (LDH) level, $\beta 2$ microglobulin (B2M) level, white blood cell count at diagnosis, Ki-67 expression and international prognostic index (IPI) (26), along with overall survival (OS), were recorded. The median follow-up time was 45.8 months (range, 4.7-109.3 months). The present retrospective study was approved by the Institutional Review Board of Tianjin Medical University Cancer Hospital.
Table I. EZH2 and HDAC1/2 expression in PTCL.

\begin{tabular}{lccc}
\hline Neoplasm & EZH2 & HDAC1 & HDAC2 \\
\hline PTCL & $53 / 82$ & $50 / 82$ & $47 / 82$ \\
PTCL-NOS & $29 / 43$ & $24 / 43$ & $25 / 43$ \\
AICL & $6 / 10$ & $5 / 10$ & $6 / 10$ \\
NK/TCL & $7 / 14$ & $8 / 14$ & $8 / 14$ \\
ALCL & $11 / 15$ & $13 / 15$ & $8 / 15$ \\
\hline
\end{tabular}

EZH2, enhancer of zeste homolog 2; HDAC, histone deacetylase; PTCL, peripheral T cell lymphoma; PTCL-NOS, PTCL not otherwise specified; AICL, angioimmunoblastic T-cell lymphoma; ALCL; anaplastic large cell lymphoma; NK/TCL, natural killer/T-cell.

The present study included 51 males $(62.2 \%)$ and 31 females (37.8\%). The mean age was 51.4 years (range, $4-81$ years) and the mean OS time was 21.6 months (range, 1.1-74.6 moths), with 21/82 patients surviving until follow-up. Stages I-II accounted for $32.9 \%(27 / 82)$ of cases and stages III-IV accounted for $67.1 \%$ (55/82). Splenomegaly and Ki-67 expression were observed in 43.9 and $64.6 \%$ of patients, respectively. There were 38 (46.3\%) low-risk and 44 (53.7\%) high-risk cases, and marrow involvement and B-symptoms were observed in 23 (28.1\%) and $53(64.6 \%)$ cases, respectively.

Immunohistochemistry. Tissues were collected and fixed in $4 \%$ formaldehyde at room temperature overnight and immunohistochemical staining was performed on $4-\mu \mathrm{m}$ formalin-fixed paraffin-embedded sections, which were provided by the Pathology Department at The Tianjin Medical University Cancer Hospital. Tissue sections were dewaxed in xylene at $60^{\circ} \mathrm{C}$ for $40 \mathrm{~min}$, rehydrated with graded alcohol and rinsed with water. Briefly, $10 \mathrm{mM}$ citrate buffer ( $\mathrm{pH}$ 6.0) was used for antigen retrieval at $120^{\circ} \mathrm{C}$ for $2.5 \mathrm{~min}$ followed by cooling to room temperature. Freshly prepared $3 \%$ hydrogen peroxide in methanol solution $\left(\mathrm{V}_{30 \% \text { hydrogen peroxide }}: \mathrm{V}_{\text {methanol }}=1: 9\right)$ was added and tissues were incubated in the dark at room temperature for $20 \mathrm{~min}$ to eliminate endogenous peroxidase activity. Following rinsing with phosphate-buffered saline, the slides were incubated with polyclonal rabbit anti-HDAC1 (catalog no. BS6485; 1:100 dilution; Bioworld Technology, Inc., St. Louis Park, MN, USA), polyclonal rabbit anti-HDAC2 (catalog no. 12922-3-AP; 1:200 dilution; ProteinTech Group, Inc., Chicago, IL, USA) and polyclonal rabbit anti-EZH2 (catalog no. BS90776; 1:50 dilution; Bioworld Technology, Inc.) primary antibodies overnight at $4^{\circ} \mathrm{C}$. Sections were subsequently incubated with unconjugated anti-rabbit IgG Ab secondary antibody (catalog no. TA130015; 1:200 dilution; OriGene Technologies, Inc., Beijing, China) at $37^{\circ} \mathrm{C}$ for $90 \mathrm{~min}$, and were stained with hematoxylin for $3 \mathrm{~min}$ at room temperature. Negative controls were included by omitting the primary antibody. Tissues were imaged at x200 and x400 magnification with an optical microscope. Prostate and breast cancer tissues were used as positive controls as these tissues exhibit a high expression of $\mathrm{HDAC} 1 / 2$ and $\mathrm{EZH} 2$, respectively.

Evaluation of immunohistochemistry. Antibodies against $\mathrm{HDAC} 1 / 2$ and $\mathrm{EZH} 2$ proteins were used to stain the nuclei 
Table II. Correlations between EZH2 and HDAC1/2 in four subtypes of PTCL.

\begin{tabular}{|c|c|c|c|c|c|c|c|c|c|c|}
\hline \multirow[b]{2}{*}{ Comparison } & \multicolumn{2}{|c|}{ PTCL } & \multicolumn{2}{|c|}{ PTCL-NOS } & \multicolumn{2}{|c|}{ AICL } & \multicolumn{2}{|c|}{ NK/TCL } & \multicolumn{2}{|c|}{ ALCL } \\
\hline & HDAC1 & HDAC2 & HDAC1 & HDAC2 & HDAC1 & HDAC2 & HDAC1 & HDAC2 & HDAC 1 & HDAC2 \\
\hline EZH2 & $0.007^{\mathrm{a}}$ & $0.005^{\mathrm{a}}$ & 0.068 & $<0.001^{\mathrm{a}}$ & 1.000 & 0.242 & 0.317 & 0.031 & $0.009^{\mathrm{a}}$ & 0.109 \\
\hline
\end{tabular}

${ }^{a} \mathrm{P}<0.05$. EZH2, enhancer of zeste homolog 2; HDAC, histone deacetylase; PTCL, peripheral T cell lymphoma; PTCL-NOS, PTCL not otherwise specified; ALCL, anaplastic large cell lymphoma; NK/TCL, natural killer/T-cell; AICL, angioimmunoblastic T-cell lymphoma.

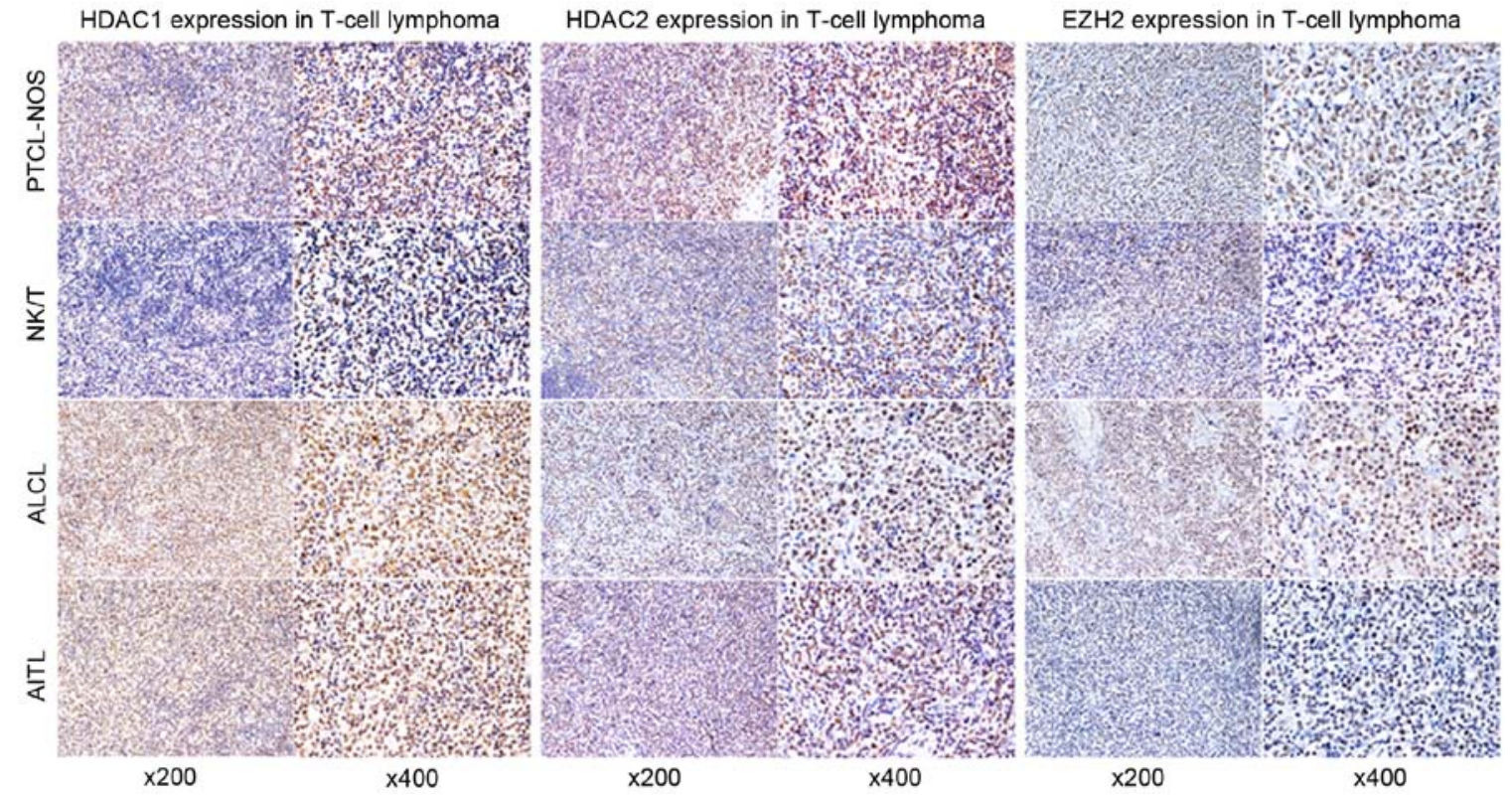

Figure 1. Representative immunohistochemical features of HDAC1 (left), HDAC2 (middle), and EZH2 (right) in PTCL-NOS, ALCL, NK/T and AITL. All images were captured at x200 and x400 magnifications. HDAC, histone deacetylase; EZH2, enhancer of zeste homolog 2; PTCL-NOS, peripheral T cell lymphoma not otherwise specified; ALCL, anaplastic large cell lymphoma; NK/T, natural killer/T-cell; AITL, angioimmunoblastic T-cell lymphoma.

of lymphoma cells. Immunohistochemical staining was interpreted based upon the following two parameters: The staining intensity and the proportion of positively-stained cells. The number of positively-stained cells was scored as follows: 0 , $<5 \% ; 1,>5 \%$ and $\leq 25 \% ; 2,>25 \%$ and $\leq 50 \%$; and $3,>50 \%$ positive cells. The intensity of positivity was scored as follows: 0 , no positivity; 1 , weak positivity; 2 , moderate positivity; and 3 , strong positivity. The values of the two scores were then multiplied with a score of $<3$ as the low expression group and a score of $\geq 3$ as the high expression group.

Survival and statistical analysis. IBM SPSS version 18.0 (SPSS, Inc., Chicago, IL, USA) was used for statistical analysis. Associations between HDAC1, HDAC2 or EZH2 expression and clinicopathological characteristics were analyzed using the a $\chi^{2}$ test. Correlations between EZH 2 and HDAC1/2 expression were analyzed using Phi coefficient analysis. Univariate OS rates were obtained using the Kaplan-Meier method and the log-rank test. OS periods were defined as the intervals between primary surgery and the last follow-up visit or mortality from any cause. The Cox proportional hazards model was used to evaluate the associations between clinicopathological factors and survival rates. The hazard ratio (HR) and associated 95\% confidence interval (CI) were calculated for each variable. $\mathrm{P}<0.05$ was considered to indicate a statistically significant difference.

\section{Results}

Correlation between EZH2 and HDACl and HDAC2 expression in PTCL. The percentages of patients expressing EZH2, HDAC1 and HDAC2 were 64.6\% (53/82), 61.0\% (50/82) and $57.3 \%$ (47/82), respectively, (staining intensity score $\geq 3$ ). Table I presents the expression of these biomarkers in PTCL, PTCL-NOS, AITL, NK/TCL and ALCL. Strong nuclear staining for EZH2, HDAC1 and HDAC2 were observed in each subtype. High EZH2 expression was observed in 29/43 PTCL-NOS (67.5\%), 6/10 AITL (60\%), 7/14 NK/TCL (50\%) and 11/15 ALCL (73.3\%) cases. High HDAC1 expression was observed in 24/43 PTCL-NOS (55.8\%), 5/10 AITL (50\%), 8/14 NK/TCL (57.1\%) and 13/15 ALCL (86.7\%) cases. High HDAC2 expression was observed in 25/43 PTCL-NOS (58.1\%), 6/10 AITL (60\%), 8/14 NK/TCL (57.1\%) and 8/15 ALCL (53.3\%) cases. Representative EZH2 and HDAC1/2 immunostaining are presented in Fig. 1. 
Table III. Correlations between EZH2/HDAC1/2 expression and the clinicopathological characteristics in PTCL.

\begin{tabular}{|c|c|c|c|c|c|c|c|c|c|}
\hline \multirow[b]{2}{*}{ Characteristic } & \multicolumn{3}{|c|}{ EZH2 expression } & \multicolumn{3}{|c|}{ HDAC 1 expression } & \multicolumn{3}{|c|}{ HDAC2 expression } \\
\hline & High & Low & P-value & High & Low & P-value & High & Low & P-value \\
\hline \multicolumn{10}{|l|}{ Sex } \\
\hline Male & $30(58.8)$ & $21(41.2)$ & 0.158 & $31(60.8)$ & $20(39.2)$ & 0.964 & $24(47.1)$ & $27(52.9)$ & $0.016^{\mathrm{b}}$ \\
\hline Female & $23(74.2)$ & $8(25.8)$ & & $19(61.3)$ & $12(38.7)$ & & $23(74.2)$ & $8(25.8)$ & \\
\hline \multicolumn{10}{|l|}{ Age, years } \\
\hline$\leq 60$ & $32(65.3)$ & $17(34.7)$ & 0.877 & $34(69.4)$ & $15(30.6)$ & 0.057 & $30(61.2)$ & $19(38.8)$ & 0.383 \\
\hline$>60$ & $21(63.6)$ & $12(36.4)$ & & $16(48.5)$ & $17(51.5)$ & & $17(51.5)$ & $16(48.5)$ & \\
\hline \multicolumn{10}{|l|}{ B-symptoms } \\
\hline Present & $39(73.6)$ & $14(26.4)$ & $0.022^{\mathrm{b}}$ & $36(67.9)$ & $17(32.1)$ & 0.081 & $31(58.5)$ & $22(41.5)$ & 0.771 \\
\hline Absent & $14(48.3)$ & $15(51.7)$ & & $14(48.3)$ & $15(51.7)$ & & $16(55.2)$ & $13(44.8)$ & \\
\hline \multicolumn{10}{|l|}{ Marrow involvement } \\
\hline Present & $18(78.3)$ & $5(21.7)$ & 0.107 & $16(69.6)$ & $7(30.4)$ & 0.319 & $17(85.0)$ & $3(15.0)$ & 0.007 \\
\hline Absent & $35(59.3)$ & $24(40.7)$ & & $34(57.6)$ & $25(42.4)$ & & $30(50.8)$ & $29(49.2)$ & \\
\hline \multicolumn{10}{|l|}{ Splenomegaly } \\
\hline Present & $21(58.3)$ & $15(41.7)$ & 0.291 & $20(55.6)$ & $16(44.4)$ & 0.373 & $22(61.1)$ & $14(38.9)$ & 0.555 \\
\hline Absent & $32(69.6)$ & $14(30.4)$ & & $30(65.2)$ & $16(34.8)$ & & $31(67.4)$ & $15(32.6)$ & \\
\hline \multicolumn{10}{|l|}{ Stage } \\
\hline I-II & $14(53.8)$ & $12(46.2)$ & 0.164 & $12(46.2)$ & $14(53.8)$ & 0.061 & $9(34.6)$ & $17(65.4)$ & $0.005^{\mathrm{a}}$ \\
\hline III-IV & $39(69.6)$ & $17(30.4)$ & & $38(67.9)$ & $18(32.1)$ & & $38(67.9)$ & $18(32.1)$ & \\
\hline \multicolumn{10}{|l|}{ IPI } \\
\hline $0-2$ & $27(60.0)$ & $18(40.0)$ & 0.333 & $23(51.1)$ & 22 (48.9) & 0.043 & $21(46.7)$ & $24(53.3)$ & $0.032^{\mathrm{b}}$ \\
\hline $3-5$ & $26(70.3)$ & $11(29.7)$ & & $27(73.0)$ & $10(27.0)$ & & $26(70.3)$ & $11(29.7)$ & \\
\hline \multicolumn{10}{|l|}{ B2M } \\
\hline$>$ Upper limit of normal & $35(76.1)$ & $11(23.9)$ & $0.014^{\mathrm{b}}$ & $32(69.6)$ & $14(30.4)$ & 0.071 & $31(67.4)$ & $15(32.6)$ & $0.037^{\mathrm{b}}$ \\
\hline Normal & $18(50.0)$ & $18(50.0)$ & & $18(50.0)$ & $18(50.0)$ & & $16(44.4)$ & $20(55.6)$ & \\
\hline \multicolumn{10}{|l|}{ LDH } \\
\hline$>$ Upper limit of normal & $40(72.7)$ & $15(27.3)$ & $0.029^{\mathrm{b}}$ & $30(61.2)$ & $19(38.8)$ & 0.813 & $34(61.8)$ & $21(38.2)$ & 0.240 \\
\hline Normal & $13(48.1)$ & $14(51.9)$ & & $14(58.3)$ & $10(41.7)$ & & $13(48.1)$ & $14(51.9)$ & \\
\hline \multicolumn{10}{|l|}{ WBC } \\
\hline$>$ Upper limit of normal & $11(44.0)$ & $14(56.0)$ & $0.010^{\mathrm{b}}$ & $12(48.0)$ & $13(52.0)$ & 0.111 & $11(44.0)$ & $14(56.0)$ & 0.106 \\
\hline Normal & $42(73.7)$ & $15(26.3)$ & & 38 (66.7) & $19(33.3)$ & & $36(63.2)$ & $21(36.8)$ & \\
\hline \multicolumn{10}{|l|}{ Ki-67 } \\
\hline$\geq 30 \%$ & $38(71.7)$ & $15(28.3)$ & 0.070 & $33(62.3)$ & $20(37.7)$ & 0.746 & $32(60.4)$ & 21 (39.6) & 0.449 \\
\hline$<30 \%$ & $15(51.7)$ & $14(48.3)$ & & $17(58.6)$ & $12(41.4)$ & & $15(51.7)$ & $14(48.3)$ & \\
\hline \multicolumn{10}{|l|}{ Pathology } \\
\hline PTCL-NOS & $26(70.3)$ & $11(29.7)$ & 0.440 & $22(59.5)$ & $15(40.5)$ & 0.212 & $26(70.3)$ & $11(29.7)$ & 0.962 \\
\hline Other & $19(79.2)$ & $5(20.8)$ & & $18(75)$ & $6(25)$ & & $17(70.8)$ & $7(29.2)$ & \\
\hline
\end{tabular}

${ }^{\mathrm{a}} \mathrm{P}<0.01,{ }^{\mathrm{b}} \mathrm{P}<0.05$. EZH2, enhancer of zeste homolog 2; HDAC, histone deacetylase; PTCL, peripheral T cell lymphoma; PTCL-NOS, PTCL not otherwise specified; IPI, international prognostic index; B2M, $\beta 2$ microglobulin; LDH, lactate dehydrogenase; WBC, white blood count.

Phi coefficient analysis demonstrated that EZH2 expression was correlated with HDAC1 and HDAC2 expression $(r=0.297$ and $r=0.306$, respectively; $\mathrm{P}<0.01)$. Of the four subtypes, the correlation between EZH2 and HDAC2 expression was only observed in patients with PTCL-NOS $(\mathrm{r}=0.517 ; \mathrm{P}<0.01)$. However, the correlation between EZH2 and HDAC1 was observed in patients with ALCL $(r=0.577$; $\mathrm{P}<0.05$ ) (Table II).
Association between EZH2, HDAC1 and HDAC2 expression and clinicopathological characteristics in PTCL. In patients with PTCL, high EZH2 expression was significantly associated with the presence of $\mathrm{B}$ symptoms $(\mathrm{P}=0.022)$, elevated LDH levels ( $\mathrm{P}=0.029)$, elevated B2M levels $(\mathrm{P}=0.014)$ and a high white blood cell count $(\mathrm{P}=0.010)$. High HDAC2 expression was significantly associated with sex $(\mathrm{P}=0.016)$, Marrow involvement $(\mathrm{P}=0.07)$, advanced clinical stage $(\mathrm{P}=0.005)$, a 
Table IV. Correlations between the EZH2/HDAC1/2 expression and the clinicopathological characteristics in PTCL-NOS.

\begin{tabular}{|c|c|c|c|c|c|c|c|c|c|}
\hline \multirow[b]{2}{*}{ Characteristic } & \multicolumn{3}{|c|}{ EZH2 expression } & \multicolumn{3}{|c|}{ HDAC1 expression } & \multicolumn{3}{|c|}{ HDAC2 expression } \\
\hline & High & Low & P-value & High & Low & P-value & High & Low & $\mathrm{P}$-value \\
\hline \multicolumn{10}{|l|}{ Sex } \\
\hline Male & $20(62.5)$ & $12(37.5)$ & 0.420 & $18(56.2)$ & $14(43.8)$ & 0.213 & $17(53.1)$ & 15 (46.9) & 0.434 \\
\hline Female & $9(81.8)$ & $2(18.2)$ & & $14(73.7)$ & $5(26.3)$ & & $8(72.7)$ & $3(27.3)$ & \\
\hline \multicolumn{10}{|l|}{ Age, years } \\
\hline$\leq 60$ & $15(73.7)$ & $9(26.3)$ & 0.437 & $16(66.7)$ & $8(33.3)$ & 0.107 & $14(58.3)$ & $10(41.7)$ & 0.977 \\
\hline$>60$ & $14(62.5)$ & $5(37.5)$ & & $8(42.1)$ & $11(57.9)$ & & $11(57.9)$ & $8(42.1)$ & \\
\hline \multicolumn{10}{|l|}{ B-symptoms } \\
\hline Present & $20(74.1)$ & 7 (25.9) & 0.228 & $17(63.0)$ & $10(37.0)$ & 0.220 & $16(59.3)$ & $11(40.7)$ & 0.847 \\
\hline Absent & $9(56.3)$ & $7(43.7)$ & & $7(43.8)$ & $9(56.3)$ & & $9(56.2)$ & $7(43.8)$ & \\
\hline \multicolumn{10}{|l|}{ Marrow involvement } \\
\hline Present & $12(85.7)$ & $2(14.3)$ & 0.153 & $8(57.1)$ & $6(42.9)$ & 0.903 & $11(78.6)$ & $3(21.4)$ & 0.059 \\
\hline Absent & $17(58.6)$ & $12(41.4)$ & & $16(55.2)$ & $13(44.8)$ & & $14(48.3)$ & $15(51.7)$ & \\
\hline \multicolumn{10}{|l|}{ Splenomegaly } \\
\hline Present & $11(55.0)$ & $9(45.0)$ & 0.104 & $10(50.0)$ & $10(50.0)$ & 0.474 & $9(45.0)$ & $11(55.0)$ & 0.103 \\
\hline Absent & $18(78.3)$ & $5(21.7)$ & & $14(60.9)$ & $9(39.1)$ & & $16(69.6)$ & $7(30.4)$ & \\
\hline \multicolumn{10}{|l|}{ Stage } \\
\hline I-II & $5(38.5)$ & $8(61.5)$ & $0.021^{\mathrm{b}}$ & $4(30.8)$ & $9(69.2)$ & $0.029^{\mathrm{b}}$ & $2(47.4)$ & $11(52.6)$ & $0.000^{\mathrm{a}}$ \\
\hline III-IV & $24(80.0)$ & $6(20.0)$ & & $20(66.7)$ & $10(33.3)$ & & $23(80.9)$ & $7(19.1)$ & \\
\hline \multicolumn{10}{|l|}{ IPI } \\
\hline $0-2$ & $14(60.9)$ & $9(39.1)$ & 0.324 & $10(43.5)$ & $13(56.5)$ & 0.081 & $11(47.8)$ & $12(52.2)$ & 0.142 \\
\hline $3-5$ & $15(75.0)$ & $5(25.0)$ & & $14(70.0)$ & $6(30.0)$ & & $4(70.0)$ & $6(30.0)$ & \\
\hline \multicolumn{10}{|l|}{$\mathrm{B} 2 \mathrm{M}$} \\
\hline >Upper limit of normal & $18(75.0)$ & $6(25.0)$ & 0.235 & $18(75.0)$ & $6(25.0)$ & $0.004^{\mathrm{a}}$ & $15(62.5)$ & $9(37.5)$ & 0.515 \\
\hline Normal & $11(57.9)$ & $8(42.1)$ & & $6(31.6)$ & $13(68.4)$ & & $10(52.6)$ & $9(47.4)$ & \\
\hline \multicolumn{10}{|l|}{ LDH } \\
\hline$>$ Upper limit of normal & $20(76.9)$ & $6(23.1)$ & 0.101 & $18(69.2)$ & $8(30.8)$ & 0.028 & $16(61.5)$ & $10(38.5)$ & 0.576 \\
\hline Normal & $9(52.9)$ & $8(47.1)$ & & $6(35.3)$ & $11(64.7)$ & & $9(52.9)$ & $8(47.1)$ & \\
\hline \multicolumn{10}{|l|}{ WBC } \\
\hline$>$ Upper limit of normal & $7(50.0)$ & $7(50.0)$ & 0.177 & $7(50.0)$ & $7(50.0)$ & 0.594 & $8(57.1)$ & $6(42.9)$ & 0.927 \\
\hline Normal & $22(78.6)$ & $7(21.4)$ & & $17(58.6)$ & $12(41.4)$ & & $17(58.6)$ & $12(41.4)$ & \\
\hline \multicolumn{10}{|l|}{ Ki-67 } \\
\hline$\geq 30 \%$ & $21(80.8)$ & $5(19.2)$ & $0.021^{\mathrm{b}}$ & $15(57.7)$ & $11(42.3)$ & 0.759 & $17(65.4)$ & $9(34.6)$ & 0.234 \\
\hline$<30 \%$ & $8(47.1)$ & $9(52.9)$ & & $9(52.9)$ & $8(47.1)$ & & $8(47.1)$ & $9(52.9)$ & \\
\hline
\end{tabular}

${ }^{\mathrm{a}} \mathrm{P}<0.01,{ }^{\mathrm{b}} \mathrm{P}<0.05$. EZH2, enhancer of zeste homolog 2; HDAC, histone deacetylase; PTCL-NOS, PTCL not otherwise specified; IPI, international prognostic index; B2M, $\beta 2$ microglobulin; LDH, lactate dehydrogenase; WBC, white blood count.

high IPI score $(\mathrm{P}=0.032)$ and elevated $\mathrm{B} 2 \mathrm{M}$ levels $(\mathrm{P}=0.037)$. High HDAC1 expression was only significantly associated with a high IPI score $(\mathrm{P}=0.043$; Table III).

In PTCL-NOS patients, high EZH2 expression was significantly associated with advanced clinical stage $(\mathrm{P}=0.021)$ and high Ki-67 expression ( $\mathrm{P}=0.021)$, while overexpression of HDAC1 was significantly associated with advanced clinical stage $(\mathrm{P}=0.029)$, elevated $\mathrm{B} 2 \mathrm{M}$ levels $(\mathrm{P}=0.004)$ and elevated LDH levels $(\mathrm{P}=0.028)$. The overexpression of HDAC2 was significantly associated with advanced clinical stage $(\mathrm{P}=0.000$; Table IV). However, the associations between high expression and low expression of these proteins were insignificant in NK/TCL patients (Table V).

Correlation between EZH2, HDAC1 and HDAC2, and survival. The median follow-up period was 45.8 months (range, 4.7-109.3 months). The 3-year OS rates of the high and low EZH2 expression PTCL groups were 28.0 and $40.2 \%$, respectively $(\mathrm{P}=0.012)$. The 3-year OS rates of the high and low HDAC2 expression groups were 16.5 and $57.0 \%$, respectively $(\mathrm{P}<0.01)$. However, the 3-year OS rates of the high and low HDAC1 expression groups exhibited had no significant differences $(\mathrm{P}>0.05)$. 
Table V. Correlations between the EZH2/HDAC1/2 expression and the clinicopathological characteristics in NK/TCL.

\begin{tabular}{|c|c|c|c|c|c|c|c|c|c|}
\hline \multirow[b]{2}{*}{ Characteristic } & \multicolumn{3}{|c|}{ EZH2 expression } & \multicolumn{3}{|c|}{ HDAC1 expression } & \multicolumn{3}{|c|}{ HDAC2 expression } \\
\hline & High & Low & P-value & High & Low & P-value & High & Low & P-value \\
\hline \multicolumn{10}{|l|}{ Sex } \\
\hline Male & $2(33.3)$ & $4(66.7)$ & 0.589 & $3(50.0)$ & $3(50.0)$ & 1.000 & $1(16.7)$ & $5(83.3)$ & $0.035^{\mathrm{a}}$ \\
\hline Female & $5(62.5)$ & $3(37.5)$ & & $5(62.5)$ & $3(37.5)$ & & $7(87.5)$ & $1(12.5)$ & \\
\hline \multicolumn{10}{|l|}{ Age, years } \\
\hline$\leq 60$ & $6(85.7)$ & $6(14.3)$ & 1.000 & $6(50.0)$ & $6(50.0)$ & 0.115 & $7(58.3)$ & $5(41.7)$ & 0.826 \\
\hline$>60$ & $1(85.7)$ & $1(14.3)$ & & $2(100.0)$ & $0(00.0)$ & & $1(50.0)$ & $1(50.0)$ & \\
\hline \multicolumn{10}{|l|}{ B-symptoms } \\
\hline Present & $6(60.0)$ & $4(40.0)$ & 0.554 & $7(70.0)$ & $3(30.0)$ & 0.348 & $6(60.0)$ & $4(40.0)$ & 1.000 \\
\hline Absent & $1(25.0)$ & $3(75.0)$ & & $1(25.0)$ & $3(75.0)$ & & $2(50.0)$ & $2(50.0)$ & \\
\hline \multicolumn{10}{|l|}{ Marrow involvement } \\
\hline Present & $2(66.7)$ & $1(33.3)$ & 1.000 & $2(66.7)$ & $1(33.3)$ & 1.000 & $2(66.7)$ & $1(33.3)$ & 1.000 \\
\hline Absent & $5(45.5)$ & $6(54.5)$ & & $6(54.5)$ & $5(45.5)$ & & $6(54.5)$ & $5(45.5)$ & \\
\hline \multicolumn{10}{|l|}{ Splenomegaly } \\
\hline Present & $3(50.0)$ & $3(50.0)$ & 1.000 & $4(66.7)$ & $2(33.3)$ & 0.938 & $5(83.3)$ & $1(16.7)$ & 0.242 \\
\hline Absent & $4(50.0)$ & $4(50.0)$ & & $4(50.0)$ & $4(50.0)$ & & $3(37.5)$ & $5(62.5)$ & \\
\hline \multicolumn{10}{|l|}{ Stage } \\
\hline I-II & $2(50.0)$ & $2(50.0)$ & 1.000 & $2(50.0)$ & $2(50.0)$ & 1.000 & $1(25.0)$ & $3(75.0)$ & 0.348 \\
\hline III-IV & $5(50.0)$ & $5(50.0)$ & & $6(60.0)$ & $4(40.0)$ & & $7(70.0)$ & $3(30.0)$ & \\
\hline \multicolumn{10}{|l|}{ IPI } \\
\hline $0-2$ & $4(50.0)$ & $4(50.0)$ & 1.000 & $5(62.5)$ & $3(37.5)$ & 1.000 & $3(37.5)$ & $5(62.5)$ & 0.242 \\
\hline $3-5$ & $3(50.0)$ & $3(50.0)$ & & $3(50.0)$ & $3(50.0)$ & & $5(83.3)$ & $1(16.7)$ & \\
\hline \multicolumn{10}{|l|}{$\mathrm{B} 2 \mathrm{M}$} \\
\hline$>$ Upper limit of normal & $6(66.7)$ & $3(33.3)$ & 0.265 & $5(55.6)$ & $4(44.4)$ & 1.000 & $6(66.7)$ & $3(33.3)$ & 0.687 \\
\hline Normal & $1(20.0)$ & $4(80.0)$ & & $3(60.0)$ & $2(40.0)$ & & $2(40.0)$ & $3(60.0)$ & \\
\hline \multicolumn{10}{|l|}{$\mathrm{LDH}$} \\
\hline$>$ Upper limit of normal & $6(60.0)$ & $4(40.0)$ & 0.554 & $5(50.0)$ & $5(50.0)$ & 0.798 & $7(70.0)$ & $3(30.0)$ & 0.348 \\
\hline Normal & $1(33.3)$ & $3(66.7)$ & & $3(75.0)$ & $1(25.0)$ & & $1(25.0)$ & $3(75.0)$ & \\
\hline \multicolumn{10}{|l|}{ WBC } \\
\hline$>$ Upper limit of normal & $0(100.0)$ & $3(00.0)$ & 0.193 & $1(33.3)$ & $2(66.7)$ & 0.778 & $0(00.0)$ & $3(100.0)$ & 0.110 \\
\hline Normal & $7(63.7)$ & $4(36.3)$ & & $7(63.6)$ & $4(36.4)$ & & $8(72.7)$ & $3(27.3)$ & \\
\hline \multicolumn{10}{|l|}{$\mathrm{Ki}-67$} \\
\hline$\geq 30 \%$ & $4(36.4)$ & $7(63.6)$ & 0.193 & $5(45.5)$ & $6(54.5)$ & 0.301 & $5(45.5)$ & $6(54.5)$ & 0.301 \\
\hline$<30 \%$ & $3(100.0)$ & $0(00.0)$ & & $3(100.0)$ & $0(00.0)$ & & $3(100.0)$ & $0(00.0)$ & \\
\hline
\end{tabular}

${ }^{\mathrm{a}} \mathrm{P}<0.05$. EZH2, enhancer of zeste homolog 2; HDAC, histone deacetylase; NK/TCL, natural killer/T cell lymphoma; IPI, international prognostic index; B2M, $\beta 2$ microglobulin; LDH, lactate dehydrogenase; WBC, white blood count

The OS rate was significantly poorer in patients with PTCL exhibiting high EZH2 and HDAC2 expression compared with those exhibiting low expression $(\mathrm{P}<0.05$; Fig. 2). This finding was also observed in the PTCL-NOS subtype ( $\mathrm{P}<0.05$; Fig. 3).

Multivariate analysis of age, sex, Ki-67 expression, IPI, clinical stage, B symptoms, marrow involvement, LDH and $\mathrm{B} 2 \mathrm{M}$ levels during diagnosis was performed. The results revealed that advanced clinical stage $(\mathrm{P}=0.024$; HR, 0.360; 95\% CI, 0.148-0.875) and HDAC2 expression $(\mathrm{P}=0.027$; HR, 0.462; 95\% CI, 0.234-0.914), but not EZH2 and HDAC1 expression, were significantly associated with a poor OS, indicating that HDAC2 may be an independent prognostic factor in PTCL (Table VI). Similar results were observed in PTCL-NOS (Table VII).

\section{Discussion}

Tumorigenesis involves gene mutation and epigenetics, which contribute to the heritable alteration of cellular biological functions. Epigenetics include DNA methylation, histone acetylation, chromatin remodeling, genetic imprinting and random chromosome $(\mathrm{X})$ inactivation. In particular, histone 

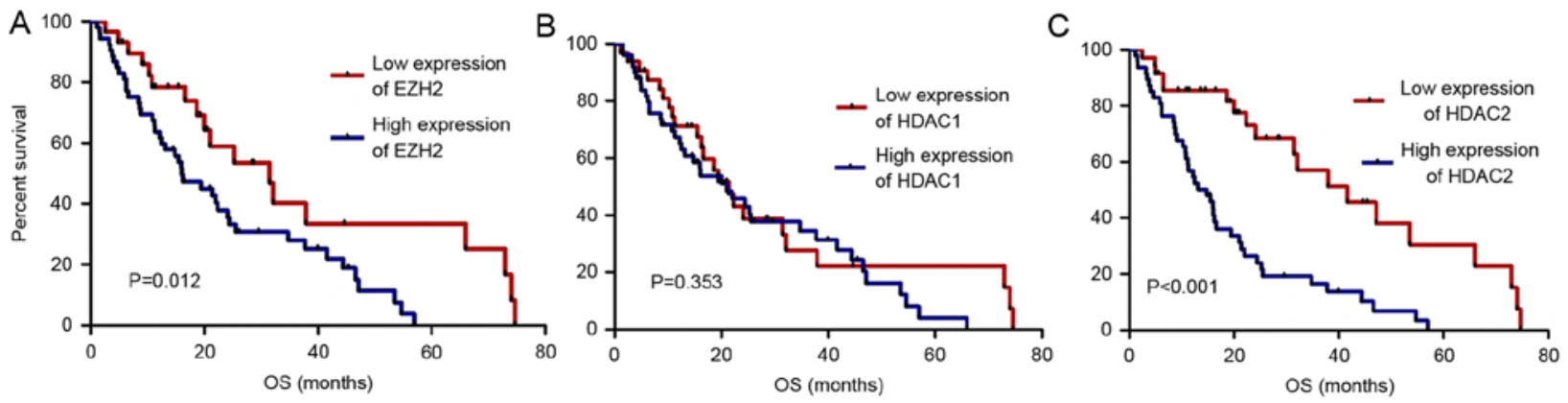

Figure 2. OS rates based upon the expression levels of EZH2 and HDAC1/2 in PTCL. (A) Significant trend towards poorer OS rates for patients with high EZH2 expression ( $\mathrm{P}=0.012)$. (C) High expression of HDAC2 was correlated with a poorer OS rate compared with low expression of HDAC2 ( $\mathrm{P}<0.001)$, which was not observed in the HDAC1 group [(B); $\mathrm{P}=0.353]$. OS, overall survival; HDAC, histone deacetylase; EZH2, enhancer of zeste homolog 2.
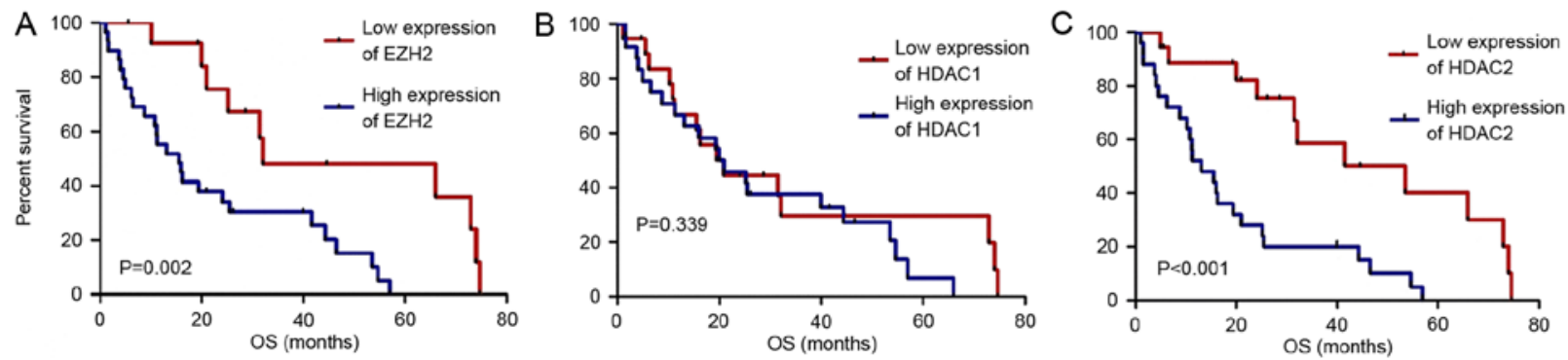

Figure 3. OS based upon the levels of EZH2 and HDAC1/2 in PTCL-NOS. (A) Significant trend towards poorer OS rates for patients with high EZH2 $(\mathrm{P}=0.002)$. (C) High expression of HDAC2 was correlated with a poorer OS rate, compared with low expression of HDAC2 ( $\mathrm{P}<0.001)$, which was not observed in the HDAC1 group [(B); $\mathrm{P}=0.339]$. OS, overall survival; HDAC, histone deacetylase; EZH2, enhancer of zeste homolog 2; PTCL-NOS, peripheral T cell lymphoma not otherwise specified.

methylation and acetylation serve critical roles in tumor development. Histone deacetylases and methyltransferases have become primary antitumorigenic targets in hematological and solid malignancies. However, few studies have been reported on the clinical significance of HDAC and EZH2 expression in PTCL.

The present study demonstrated that the patients with $\mathrm{B}$ symptoms, elevated LDH or $\beta 2-M G$ levels exhibited high EZH2 expression in their PTCL tissues, and that these factors led to a poorer OS $(\mathrm{P}<0.05)$. Based upon analysis of the pathological subtypes of PTCL, high EZH2 expression was significantly associated with advanced clinical stage and high Ki-67 expression in PTCL-NOS. The enzymatic hyperactivity of EZH2 has previously been observed in a variety of hematological malignancies, including diffused large B-cell lymphoma, follicular lymphoma, mantle cell lymphoma (27), T-lymphoblastic lymphoma (28) and adult T-cell leukemia/lymphoma (29). The results of the present study were consistent with those of previous reports that EZH2 expression is also associated with an aggressive clinical outcome (30). The results of the present study suggested that EZH2 may serve as a target for anticancer therapy in PTCL and that further research on the mechanisms of EZH2 is warranted.

Furthermore, the present study revealed that HDAC expression was associated with EZH2 expression in PTCL, which was consistent with the results of a previous study (31). HDAC1, HDAC2 and EZH2 serve important roles in DNA repair by regulating the dynamic balance between $\mathrm{H} 3 \mathrm{~K} 27 \mathrm{ac}$ and H3K27me3. This balance may be disturbed by DNA damage, resulting in tumorigenesis $(31,32)$.
The catalysis of HDAC1 and HDAC2 contributes to the reduction in $\mathrm{H} 3 \mathrm{~K} 27 \mathrm{ac}$ levels, resulting in further methylation of H3K27 by EZH2. C-myc, an important transcription regulatory factor, regulates $>70 \%$ of gene expression, including that of EZH2. C-myc upregulates the expression of EZH2 by modulating the special microRNAs in B-cell lymphoma cells (33). C-myc is overexpressed in several subsets of T-cell lymphoma (30). Therefore, several unknown associations may exist between expression of HDACs and EZH2 in PTCL.

HDACs render the gene expression profiling aberrant by deacetylating either histone or transcription factors (34-36). The US Food and Drug Administration has approved four HDAC inhibitors (HDACIs) for the treatment of cutaneous T-cell lymphoma, and the clinical application of HDACIs in other subtypes of T-cell lymphoma has received increasing attention. Based upon the results of clinical trials, three HDACIs have received conditional marketing authorization for the treatment of adult patients with relapsed or refractory aggressive PTCL. However, the clinical significance of HDAC expression in PTCL is poorly understood.

The present study demonstrated that high expression of HDAC2 frequently occurred in PTCL patients with adverse clinicopathological characteristics, including advanced clinical stage, high IPI score and elevated B2M $(\mathrm{P}<0.05)$. However, for PTCL subtype analysis, high HDAC2 expression was only associated with the clinical stage in patients with PTCL-NOS, and was only associated with patient sex in patients with NK/TCL. In addition, in patients with the PTCL-NOS subtype, high HDAC2 expression resulted in a shorter OS time than that in those exhibiting a low HDAC2 expression $(\mathrm{P}<0.05)$. This 
Table VI. Multivariate analysis of overall survival in PTCL.

\begin{tabular}{|c|c|c|}
\hline \multirow[b]{2}{*}{ Factor } & \multicolumn{2}{|c|}{ Overall survival } \\
\hline & OR $(95 \% \mathrm{CI})$ & P-value \\
\hline \multicolumn{3}{|l|}{$\mathrm{LDH}$} \\
\hline $\begin{array}{l}\text { Upper limit of } \\
\text { normal vs. normal }\end{array}$ & $0.574(0.272-1.211)$ & 0.145 \\
\hline $\begin{array}{l}\text { B2M } \\
\text { Upper limit of } \\
\text { normal vs. normal }\end{array}$ & $0.631(0.344-1.159)$ & 0.138 \\
\hline $\begin{array}{l}\text { IPI } \\
0-2 \text { vs. } 3-5\end{array}$ & $1.170(0.535-2.559)$ & 0.694 \\
\hline $\begin{array}{l}\text { Ki-67 } \\
>31 \% \text { vs. } \leq 30 \%\end{array}$ & $0.556(0.273-1.131)$ & 0.105 \\
\hline $\begin{array}{l}\text { Clinical staging } \\
\text { III-IV vs. I-II }\end{array}$ & $0.360(0.148-0.875)$ & $0.024^{\mathrm{a}}$ \\
\hline $\begin{array}{l}\text { Presence of B sympto } \\
\text { Present vs. absent }\end{array}$ & $0.642(0.342-1.207)$ & 0.169 \\
\hline $\begin{array}{l}\text { Marrow involvement } \\
\text { Present vs. absent }\end{array}$ & $0.883(0.480-1.627)$ & 0.690 \\
\hline $\begin{array}{l}\text { Sex } \\
\text { Female vs. male }\end{array}$ & $0.805(0.450-1.440)$ & 0.456 \\
\hline $\begin{array}{l}\text { Age } \\
>60 \text { vs. } \leq 60\end{array}$ & 1.039 (0.560-1.929) & 0.902 \\
\hline $\begin{array}{l}\text { Expression of HDAC } \\
\text { Low expression vs. } \\
\text { high expression }\end{array}$ & $0.462(0.234-0.914)$ & $0.027^{\mathrm{a}}$ \\
\hline
\end{tabular}

${ }^{\mathrm{a}} \mathrm{P}<0.05$. PTCL, peripheral $\mathrm{T}$ cell lymphoma; OR, odds ratio; $\mathrm{CI}$, confidence interval; LDH, lactate dehydrogenase; B2M, $\beta 2$ microglobulin; IPI, international prognostic index; HDAC2, histone deacetylase 2 .

association was observed in all PTCL patients. According to the results of the present study, HDAC2 may be a possible prognostic marker in patients with PTCL, particularly in those with the PTCL-NOS subtype. This phenomenon was not observed in the other subtypes of PTCL. One possible reason for this is the small number of patients enrolled in the present study $(37,38)$. Therefore, further studies on assessing the clinical significance of HDAC2 were required in all PTCL subtypes.

In conclusion, the present study observed that PTCL patients with high expression of EZH2 and HDAC2 usually exhibit a poorer prognosis, and that HDAC2 may be a prognostic marker in PTCL, particularly for patients with the PTCL-NOS subtype.

\section{Acknowledgements}

Not applicable.

\section{Funding}

The present study was supported by the Natural Science Foundation of China (grant nos. 81770213 and 81402945), Tianjin
Table VII. Multivariate analysis of overall survival in PTCL-NOS.

\begin{tabular}{|c|c|c|}
\hline \multirow[b]{2}{*}{ Factor } & \multicolumn{2}{|c|}{ Overall survival } \\
\hline & OR $(95 \% \mathrm{CI})$ & P-value \\
\hline \multicolumn{3}{|l|}{ LDH } \\
\hline $\begin{array}{l}\text { Upper limit of } \\
\text { normal vs. normal }\end{array}$ & $0.649(0.253-1.665)$ & 0.368 \\
\hline \multicolumn{3}{|l|}{$\mathrm{B} 2 \mathrm{M}$} \\
\hline $\begin{array}{l}\text { Upper limit of } \\
\text { normal vs. normal }\end{array}$ & $0.633(0.213-1.879)$ & 0.410 \\
\hline \multicolumn{3}{|l|}{ IPI } \\
\hline $0-2$ vs. $3-5$ & $1.012(0.300-3.411)$ & 0.985 \\
\hline \multicolumn{3}{|l|}{$\mathrm{Ki}-67$} \\
\hline$>31 \%$ vs. $\leq 30 \%$ & $0.476(0.175-1.294)$ & 0.146 \\
\hline \multicolumn{3}{|l|}{ Clinical staging } \\
\hline III-IV vs. I-II & $0.444(0.119-1.653)$ & 0.226 \\
\hline \multicolumn{3}{|c|}{ Presence of B symptoms } \\
\hline Present vs. absent & $0.468(0.185-1.185)$ & 0.109 \\
\hline \multicolumn{3}{|l|}{ Marrow involvement } \\
\hline Present vs. absent & $0.640(0.274-1.495)$ & 0.303 \\
\hline \multicolumn{3}{|l|}{ Sex } \\
\hline Female vs. male & $0.418(0.162-1.079)$ & 0.072 \\
\hline \multicolumn{3}{|l|}{ Age } \\
\hline$>60$ vs. $\leq 60$ & $0.973(0.418-2.265)$ & 0.949 \\
\hline \multicolumn{3}{|l|}{ Expression of HDAC2 } \\
\hline $\begin{array}{l}\text { Low expression vs. } \\
\text { high expression }\end{array}$ & $2.990(1.102-8.112)$ & $0.032^{\mathrm{a}}$ \\
\hline
\end{tabular}

${ }^{\text {aP }}<0.05$. PTCL-NOS, PTCL not otherwise specified; OR, odds ratio; CI, confidence interval; LDH, lactate dehydrogenase; B2M, $\beta 2$ microglobulin; IPI, international prognostic index; HDAC2, histone deacetylase 2.

Medical University Cancer Institute and Hospital Foundation (grant no. 1504) and the Key Research Projects of Tianjin Municipal Health Bureau (grant nos. 2015KZ081 and 15KG145).

\section{Availability of data and materials}

The datasets used and/or analyzed during the current study are available from the corresponding author on reasonable request.

\section{Authors' contributions}

HZ and XW designed the study. HZ, HL, XJ and XW acquired and analyzed the data, drafted the manuscript. GH, LK, TZ, LL, YP, QZ, BM, XW and HW acquired the data and critically revised the manuscript. All authors read and approved the final manuscript, and are accountable for all aspects of the study.

\section{Ethics statement and consent to participate}

The present retrospective study was approved by the Institutional Review Board of Tianjin Medical University Cancer Hospital. 


\section{Patient consent for publication}

Not applicable.

\section{Competing interests}

The authors declare that they have no competing interests.

\section{References}

1. Cai MY, Hou JH, Rao HL, Luo RZ, Li M, Pei XQ, Lin MC, Guan XY, Kung HF, Zeng YX and Xie D: High expression of $\mathrm{H} 3 \mathrm{~K} 27 \mathrm{me} 3$ in human hepatocellular carcinomas correlates closely with vascular invasion and predicts worse prognosis in patients. Mol Med 17: 12-20, 2011.

2. He LR, Liu MZ, Li BK, Rao HL, Liao YJ, Guan XY, Zeng YX and Xie D: Prognostic impact of H3K27me3 expression on locoregional progression after chemoradiotherapy in esophageal squamous cell carcinoma. BMC Cancer 9: 461, 2009.

3. Liu J, Li Y, Liao Y, Mai S, Zhang Z, Liu Z, Jiang L, Zeng Y, Zhou F and Xie D: High expression of H3K27me3 is an independent predictor of worse outcome in patients with urothelial carcinoma of bladder treated with radical cystectomy. Biomed Res Int 2013: 390482, 2013.

4. Sabattini E, Bacci F, Sagramoso C and Pileri S: WHO classification of tumours of haematopoietic and lymphoid tissues in 2008 An overview. Pathologica 102: 83-87, 2010.

5. Chen X, Song N, Matsumoto K, Nanashima A, Nagayasu T, Hayashi T, Ying M, Endo D, Wu Z and Koji T: High expression of trimethylated histone $\mathrm{H} 3$ at lysine 27 predicts better prognosis in non-small cell lung cancer. Int J Oncol 43: 1467-1480, 2013.

6. Wei Y, Xia W, Zhang Z, Liu J, Wang H, Adsay NV, Albarracin C, Yu D, Abbruzzese JL, Mills GB, et al: Loss of trimethylation at lysine 27 of histone $\mathrm{H} 3$ is a predictor of poor outcome in breast, ovarian, and pancreatic cancers. Mol Carcinog 47: 701-706, 2008.

7. Bassett SA and Barnett MP: The role of dietary histone deacetylases (HDACs) inhibitors in health and disease. Nutrients 6 : 4273-4301, 2014

8. Strahl BD and Allis CD: The language of covalent histone modifications. Nature 403: 41-45, 2000

9. Bannister AJ and Kouzarides T: Regulation of chromatin by histone modifications. Cell Res 21: 381-395, 2011.

10. Glozak MA and Seto E: Histone deacetylases and cancer. Oncogene 26: 5420-5432, 2007.

11. Smith KT and Workman JL: Histone deacetylase inhibitors: Anticancer compounds. Int J Biochem Cell Biol 41: 21-25, 2009.

12. Weichert W, Denkert C, Noske A, Darb-Esfahani S, Dietel M, Kalloger SE, Huntsman DG and Köbel M: Expression of class I histone deacetylases indicates poor prognosis in endometrioid subtypes of ovarian and endometrial carcinomas. Neoplasia 10: 1021-1027, 2008

13. Halkidou K, Gaughan L, Cook S, Leung HY, Neal DE and Robson CN: Upregulation and nuclear recruitment of HDAC1 in hormone refractory prostate cancer. Prostate 59: 177-189, 2004.

14. Weichert W, Röske A, Niesporek S, Noske A, Buckendahl AC, Dietel M, Gekeler V, Boehm M, Beckers T and Denkert C: Class I histone deacetylase expression has independent prognostic impact in human colorectal cancer: Specific role of class I histone deacetylases in vitro and in vivo. Clin Cancer Res 14: 1669-1677, 2008

15. Weichert W, Röske A, Gekeler V, Beckers T, Ebert MP, Pross M, Dietel M, Denkert C and Röcken C: Association of patterns of class I histone deacetylase expression with patient prognosis in gastric cancer: A retrospective analysis. Lancet Oncol 9: 139-148, 2008

16. Wang W, Gao J, Man XH, Li ZS and Gong YF: Significance of DNA methyltransferase-1 and histone deacetylase-1 in pancreatic cancer. Oncol Rep 21: 1439-1447, 2009.

17. Fritzsche FR, Röske A, Gekeler V, Beckers T, Stephan C, Jung K, Scholman K, Denkert C, Dietel M and Kristiansen G: Class I histone deacetylases 1,2 and 3 are highly expressed in renal cel cancer. BMC Cancer 8: 381, 2008.

18. Marquard L, Gjerdrum LM, Christensen IJ, Jensen PB, Sehested M and Ralfkiaer E: Prognostic significance of the therapeutic targets histone deacetylase 1, 2,6 and acetylated histone H4 in cutaneous T-cell lymphoma. Histopathology 53: 267-277, 2008
19. Marquard L, Poulsen CB, Gjerdrum LM, de Nully Brown P, Christensen IJ, Jensen PB, Sehested M, Johansen P and Ralfkiaer E: Histone deacetylase 1, 2, 6 and acetylated histone H4 in B- and T-cell lymphomas. Histopathology 54: 688-698, 2009.

20. Swigut T and Wysocka J: H3K27 demethylases, at long last. Cell 131: 29-32, 2007

21. Margueron R and Reinberg D: The Polycomb complex PRC2 and its mark in life. Nature 469: 343-349, 2011.

22. Cao R, Wang L, Wang H, Xia L, Erdjument-Bromage H, Tempst P, Jones RS and Zhang Y: Role of histone H3 lysine 27 methylation in Polycomb-group silencing. Science 298: 1039-1043, 2002.

23. Simon JA and Lange CA: Roles of the EZH2 histone methyltransferase in cancer epigenetics. Mutat Res 647: 21-29, 2008.

24. Velichutina I, Shaknovich R, Geng HM, Johnson NA, Gascoyne RD, Melnick AM and Elemento O: EZH2-mediated epigenetic silencing in germinal center B cells contributes to proliferation and lymphomagenesis. Blood 116: 5247-5255, 2010.

25. Musshoff K and Schmidt-Vollmer H: Proceedings: Prognosis of non-Hodgkin's lymphomas with special emphasis on the staging classification. Z Krebsforsch Klin Onkol Cancer Res Clin Oncol 83: 323-341, 1975.

26. Ziepert M, Hasenclever D, Kuhnt E, Glass B, Schmitz N, Pfreundschuh M and Loeffler M: Standard International prognostic index remains a valid predictor of outcome for patients with aggressive CD20+ B-cell lymphoma in the rituximab era. J Clin Oncol 28: 2373-2380, 2010.

27. Score J, Hidalgo-Curtis C, Jones AV, Winkelmann N, Skinner A, Ward D, Zoi K, Ernst T, Stegelmann F, Döhner K, et al: Inactivation of polycomb repressive complex 2 components in myeloproliferative and myelodysplastic/myeloproliferative neoplasms. Blood 119: 1208-1213, 2012.

28. Ntziachristos P, Tsirigos A, Van Vlierberghe P, Nedjic J, Trimarchi T, Flaherty MS, Ferres-Marco D, da Ros V, Tang Z, Siegle J, et al: Genetic inactivation of the polycomb repressive complex 2 in T cell acute lymphoblastic leukemia. Nat Med 18: 298-301, 2012.

29. Simon C, Chagraoui J, Krosl J, Gendron P, Wilhelm B, Lemieux S, Boucher G, Chagnon P, Drouin S, Lambert R, et al: A key role for EZH2 and associated genes in mouse and human adult T-cell acute leukemia. Genes Dev 26: 651-656, 2012.

30. Shi M, Shahsafaei A, Liu C, Yu H and Dorfman DM: Enhancer of zeste homolog 2 is widely expressed in T-cell neoplasms, is associated with high proliferation rate and correlates with MYC and pSTAT3 expression in a subset of cases. Leuk Lymphoma 56: 2087-2091, 2015.

31. Johnson DP, Spitz GS, Tharkar S, Quayle SN, Shearstone JR, Jones S, McDowell ME, Wellman H, Tyler JK, Cairns BR, et al: HDAC1,2 inhibition impairs EZH2- and BBAP-mediated DNA repair to overcome chemoresistance in EZH2 gain-of-function mutant diffuse large B-cell lymphoma. Oncotarget 6: 4863-4887, 2015.

32. Chou DM, Adamson B, Dephoure NE, Tan X, Nottke AC, Hurov KE, Gygi SP, Colaiácovo MP and Elledge SJ: A chromatin localization screen reveals poly (ADP ribose)-regulated recruitment of the repressive polycomb and NuRD complexes to sites of DNA damage. Proc Natl Acad Sci USA 107: 18475-18480, 2010.

33. Zappasodi R, Cavanè A, Iorio MV, Tortoreto $M$, Guarnotta C, Ruggiero G, Piovan C, Magni M, Zaffaroni N, Tagliabue E, et al: Pleiotropic antitumor effects of the pan-HDAC inhibitor ITF2357 against c-Myc-overexpressing human B-cell non-Hodgkin lymphomas. Int J Cancer 135: 2034-2045, 2014.

34. Hess-Stumpp H: Histone deacetylase inhibitors and cancer: From cell biology to the clinic. Eur J Cell Biol 84: 109-121, 2005.

35. Mariadason JM: HDACs and HDAC inhibitors in colon cancer. Epigenetics 3: 28-37, 2008.

36. Barneda-Zahonero $\mathrm{B}$ and Parra M: Histone deacetylases and cancer. Mol Oncol 6: 579-589, 2012.

37. Xie W, Hu K, Xu F, Zhou D, Huang W, He J, Shi J, Luo Y, Zhang J, Lin M, et al: Significance of clinical factors as prognostic indicators for patients with peripheral T-cell non-Hodgkin lymphoma: A retrospective analysis of 252 cases. Mol Clin Oncol 1: 911-917, 2013.

38. Gallamini A, Stelitano C, Calvi R, Bellei M, Mattei D, Vitolo U, Morabito F, Martelli M, Brusamolino E, Iannitto E, et al: Peripheral T-cell lymphoma unspecified (PTCL-U): A new prognostic model from a retrospective multicentric clinical study. Blood 103: 2474-2479, 2004. 